\title{
High intensity x-ray diffraction in transmission mode employing an analog of Poisson's spot
}

\author{
Paul Evans, ${ }^{1, a)}$ Keith Rogers, ${ }^{2}$ Jer Chan, ${ }^{1}$ Joseph Rogers, ${ }^{2}$ and Anthony Dicken ${ }^{2}$ \\ ${ }^{1}$ The Imaging Science Group, School of Science and Technology, Nottingham Trent University, Nottingham, \\ Notts NG11 8NS, United Kingdom \\ ${ }^{2}$ Cranfield University, Cranfield, Bedfordshire SN6 8LA, United Kingdom
}

(Received 21 May 2010; accepted 19 October 2010; published online 15 November 2010)

\begin{abstract}
Poisson's spot is a diffraction phenomenon producing an intensity maximum at the center of the geometric shadow of circular opaque objects. In an analog of the Poisson spot experiment, we show that a tubular cone of $\mathrm{x}$-rays incident upon a crystalline sample produces diffraction spots or foci, corresponding to Bragg maxima within a transmission shadow. We discuss the beam geometry and the intensity gain recorded at the foci in transmission mode. We describe the geometric growth and decay of the foci over a linear axis with the aid of a movie sequence synchronized with the plotting of a diffractogram. The mean signal of a small central area in each successive camera image provides the intensity data for the diffractogram. (C) 2010 American Institute of Physics. [doi:10.1063/1.3514235]
\end{abstract}

The characterization and measurement of crystallographic structure are of fundamental importance in many branches of science. Angular dispersive x-ray diffraction ${ }^{1}$ employed routinely for such analysis dominates this field. However, the coherently scattered or diffracted x-ray signatures are weaker, by orders of magnitude, in comparison with the interrogating x-ray beam. State of the art commercially available powder diffractometers may employ highly sensitive large-area detectors with high quantum efficiency and low noise operating over relatively long integration periods. ${ }^{2}$ This conventional approach is not ideal for the development of scanning techniques and direct imaging applications, which would benefit from higher intensity signals, reduced integration periods, and converging diffracted beams. In this paper we report examples of diffraction image sequences, which demonstrate the growth and collapse of $\mathrm{x}$-ray foci along the symmetry axis of a tubular interrogating x-ray beam. ${ }^{3}$ Our approach embodies an analog, employing x-rays, of the optical phenomenon that produces an intensity maximum, known as Poisson's spot, ${ }^{4}$ at the center of the geometric shadow of circular opaque objects. In common with short wavelength Poisson spot techniques employing $\mathrm{x}$-ray zone plates $^{5}$ or molecular beams ${ }^{6}$ we measure the relative intensity of diffraction maxima at the center of a circular geometric shadow. Unlike these techniques, we employ an annular zone, defined at the intersection of a tubular cone of $\mathrm{x}$-rays and a crystalline sample, enabling constructive on-axis interference to form intense spots or foci. The relative intensity and distribution of the foci correspond to the Bragg maxima determined by the crystalline structure of the sample.

Consideration of diffraction patterns composed of individual Debye ring contributions hypothesizes the formation of a Poisson spot analog in Fig. 1. A tubular cone of x-rays with its symmetry axis incident normally upon a planar polycrystalline sample and image plane (positioned on the transmission side of the sample) will produce a continuum of relatively inclined Debye cones resulting in planar patterns composed of elliptical rings. The resultant circular termini

${ }^{\text {a)} E l e c t r o n i c ~ m a i l: ~ p a u l . e v a n s @ n t u . a c . u k . ~}$ and spot intensity fluctuations are due to rotational symmetry at the image plane. The opening angle of the Debye cones is determined by Bragg's condition, $\lambda=2 d \sin \theta$, where $\lambda$ is the wavelength of the incident wave, $d$ is the spacing between the planes in the atomic lattice, and $\theta$ is the angle between the incident ray and the scattering planes.

Our experiment setup, in the photograph in Fig. 2 and schematic in Fig. 3, enables a tubular cone of x-rays, using a wavelength of $0.7107 \AA$, to be normally incident upon a planar, $0.18 \mathrm{~mm}$ thick, polycrystalline sample of aluminum oxide. The annular illuminated portion of the sample had an outer radius of $9.64 \mathrm{~mm}$ and an inner radius of $9.38 \mathrm{~mm}$ (mean radius, $R_{S}=9.51 \mathrm{~mm}$ ). The sample and x-ray source remained in fixed positions and separated by $150 \mathrm{~mm}$ throughout the experiment. A thermoelectrically cooled camera employing a charge-coupled device camera imager composed of $1024 \times 1024$ pixels, with each pixel $13 \mu \mathrm{m}$ in size, was coupled to a $\mathrm{Gd}_{2} \mathrm{O}_{2} \mathrm{~S}$ : Tb phosphor screen configured normal to the symmetry axis. The camera was mounted on a stepper motor translation stage and captured a sequence of 820 images, each integrated over a 45 s period with the im-

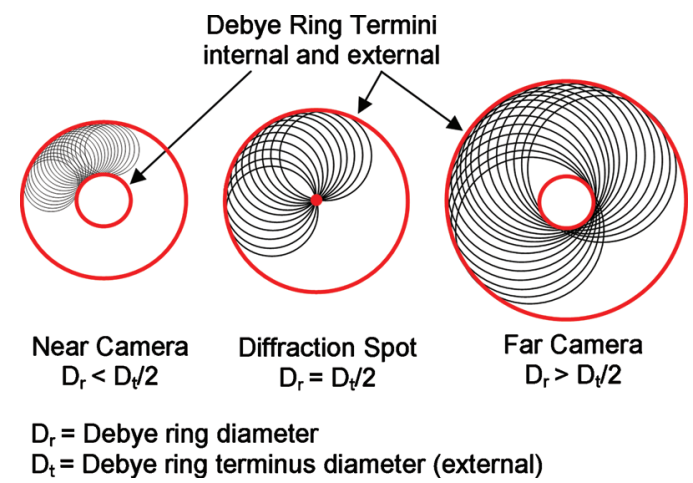

FIG. 1. (Color) The discretized representation of a continuum of Debye rings forming external and internal termini together with a Poisson spot analog at the imager. The internal terminus at $D_{r}<D_{t} / 2$ converges or collapses, with increasing imager to sample separation, to form a diffraction spot at $D_{r}=D_{t} / 2$; a further increase in separation produces a diverging or expanding circular terminus described by the pattern at $D_{r}>D_{t} / 2$. 


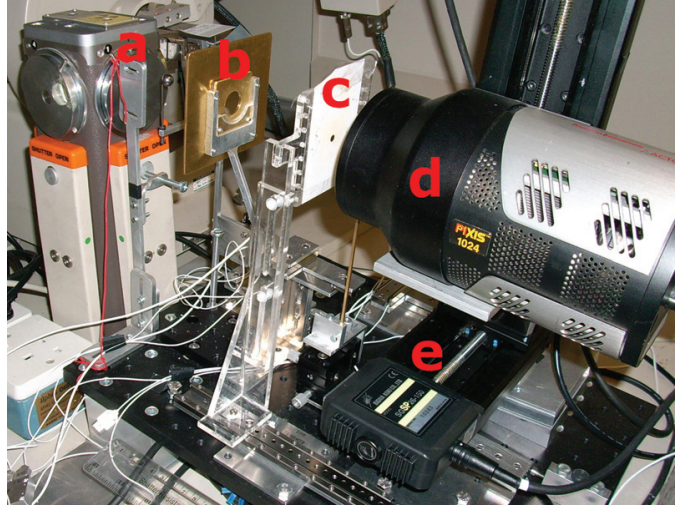

FIG. 2. (Color) Experiment apparatus. (a) X-ray port, (b) annular slit collimator, (c) sample, (d) camera, and (e) translation stages.

ager cooled to $233 \mathrm{~K}$, at $0.1 \mathrm{~mm}$ increments along an $82 \mathrm{~mm}$ component (starting at $Z=10 \mathrm{~mm}$ and ending at $Z=92 \mathrm{~mm}$ ) of the symmetry axis. The resultant diffraction images were quantized to 16 bits, representing 65536 possible gray levels, by the camera. An example of 36 images forms the thumbnail matrix of Fig. 4. A diffractogram relating the intensity of a central, approximately circular area of 80 pixels throughout the image stack against $2 \theta$ is presented with $h k l$ peak notation in Fig. 5. The mathematical equation, assuming that the tubular beam had ideally thin walls, for the approximate calculation of $2 \theta$ is given by

$$
2 \theta=\varphi+\tan ^{-1}\left(R_{s} / Z\right),
$$

where $\varphi$ is the primary $x$-ray tubular cone half angle and $Z$ is the separation between the sample and the Poisson spot along the symmetry axis, as described in Fig. 3. A software program automatically extracted and computed the mean intensity of the nominally central area for each of the diffraction images. The sampled area comprised 80 pixels to accommodate the finite size of the spot, together with any relatively small lateral shift in the incident spot positions on the imager, due to nonperfect alignment of the apparatus. The resultant diffractogram also includes a plot recorded using a pencil beam, under identical experiment conditions as the tubular cone. The pencil beam was produced by blocking the majority of the annular primary $\mathrm{x}$-rays to illuminate an

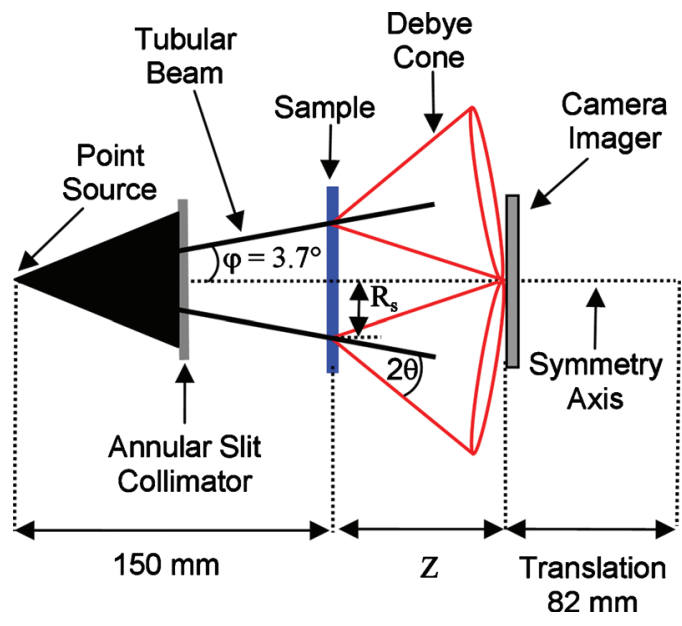

FIG. 3. (Color) Schematic depicting the experiment setup employing a tubular cone of x-rays incident upon a planar polycrystalline sample to enable a camera to capture diffraction images along the symmetry axis.

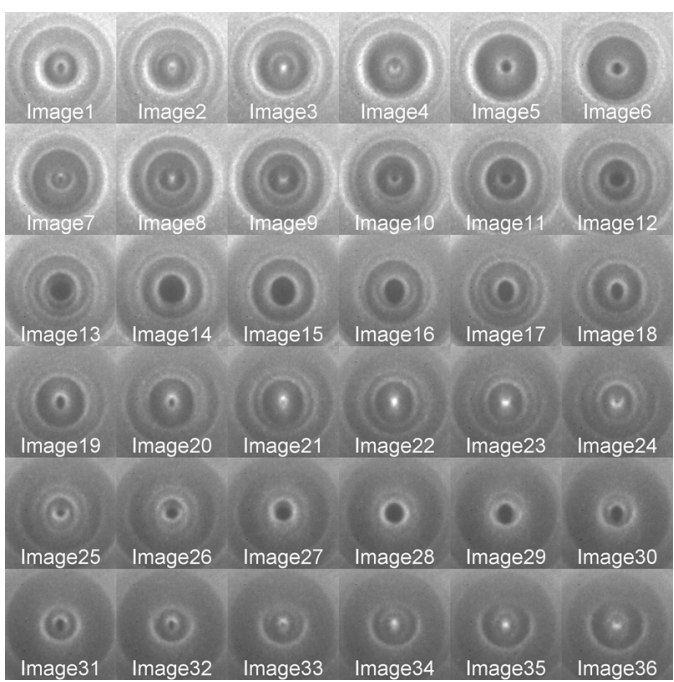

FIG. 4. An image sequence demonstrating the formation of four Poisson spot analogs extracted from a master sequence of 820 images, obtained at $0.1 \mathrm{~mm}$ increments, along the symmetry axis. Image 1 corresponds to a 21.8 $\mathrm{mm}$ separation from the aluminum oxide sample, with the remainder of the example sequence made up of every fifth image (or $0.5 \mathrm{~mm}$ step) to complete a set of 36 images. The crystallographic planes responsible for the diffraction spots apparent in images $3,8,22$, and 35 are identified by their Miller indices (116), (024), (113), and (110), respectively.

approximately rectangular $0.27 \mathrm{~mm} \times 1.29 \mathrm{~mm}$ area on the sample. This approach conserves the geometric properties of the resultant Debye rings to maintain consistent angular, $2 \theta$, resolution produced by the pencil beam and by each "element" of the tubular cone. The tubular beam foci, simultaneously produced along the symmetry axis, realize an intensity gain in comparison to the "single" Debye rings produced by the pencil beam. The gain is proportional to the ratio of the cross sectional areas of the annular x-ray beam to the pencil beam, the uniformity of the intensity of the annular footprint, and the preferred distribution of crystallographic orientations within the sample, e.g., peak 300, see Fig. 5, had a gain of 18.33. A more detailed comparison between con-

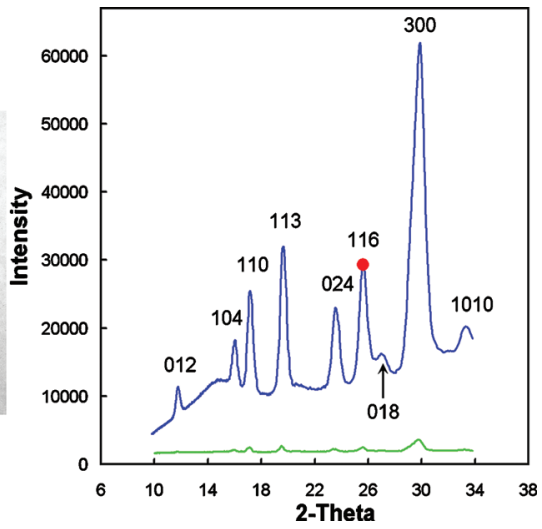

FIG. 5. (Color) A transmission mode diffractogram obtained by sampling a central, 80 pixel, area throughout the 820 image sequence to provide an angular range in 2-theta space from approximately $10^{\circ}$ to $34^{\circ}$. The profile in blue color is produced by the Poisson technique and the profile in green color is produced by a pencil beam approach. For demonstration purposes the synchronization of the plotting of a limited set of 38 data points, including one for each of the 36 images presented in Fig. 4, over a 2-theta range from approximately $17^{\circ}$ to $27^{\circ}$, is highlighted by a moving dot in red color This movie sequence demonstrates the agreement between the Poisson spot images and the expected Bragg maxima for an aluminum oxide sample (enhanced online). [URL: http://dx.doi.org/10.1063/1.3514235.1] 
ventionally derived reflection mode data published for example, in the International Centre for Diffraction Data powder diffraction databases and the transmission Poisson technique, would require the application of modified Lorentz, absorption, and preferred orientation corrections, which have not been applied to the data presented in this paper.

We conclude that appropriately designed tubular beam geometry may increase the intensity of the diffraction signals by orders of magnitude in comparison with those measured conventionally with a pencil beam. In addition, the tubular geometry integrates diffraction signatures through azimuthal angles to interrogate simultaneously sectors of reciprocal space, which could help mitigate the effects of preferred orientation. This latter point coupled with use of extended sample illumination to effect an amplification of the intensity of inherently weak diffraction signals make our technique a promising candidate for high specificity and high speed molecular scanning applications.
Our work is funded under the Innovative Research Call in Explosives and Weapons Detection, a cross-government program sponsored by a number of U.K. government departments and agencies under the CONTEST strategy and the Engineering and Physical Sciences Research Council (EPSRC) (Grant Nos. EP/F017596/1 and EP/F017804/1).

${ }^{1}$ R. Jenkins and R. L. Snyder, Introduction to X-Ray Powder Diffractometry (Wiley, New York, 1996).

${ }^{2}$ F. Zamponi, Z. Ansari, M. Woerner, and T. Elsaesser, Opt. Express 18, 947 (2010).

${ }^{3}$ K. Rogers, P. Evans, J. Rogers, J. W. Chan, and A. Dicken, J. Appl. Crystallogr. 43, 264 (2010)

${ }^{4}$ W. R. Kelly, E. L. Shirley, A. L. Migdall, S. V. Polyakov, and K. Hendrix, Am. J. Phys. 77, 713 (2009).

${ }^{5}$ E. Hecht, Optics, 2nd ed. (Addison-Wesley, Reading, 1987).

${ }^{6}$ T. Reisinger, A. A. Patel, H. Reingruber, K. Fladischer, W. E. Ernst, G. Bracco, H. I. Smith, and B. Holst, Phys. Rev. A 79, 053823 (2009). 